\title{
Editorial: Maker Education: Opportunities and Challenges
}

\author{
Janette M. Hughes ${ }^{1 *}$ and Kristiina Kumpulainen ${ }^{2}$ \\ ${ }^{1}$ Ontario Tech University, Oshawa, ON, Canada, ${ }^{2}$ University of Helsinki, Helsinki, Finland
}

Keywords: maker education, makerspaces, maker pedagogies, steam, stem

\section{Editorial on the Research Topic}

\section{Maker Education: Opportunities and Challenges}

This robust collection of articles focuses on makers and maker educators in K-12, as well as makerspaces, maker programs and maker pedagogies both in and out of school in four different countries (Canada, Finland, Iceland and the United States). The eight research studies offer insights into several key themes in developing maker culture in a variety of settings through different research methodologies.

Makerspaces and maker education have typically been associated with STEM (Science, Technology, Engineering and Math) subjects, where there has been an emphasis on the use of digital technologies (high, medium and low-tech) and engineering design processes to teach science, math and computational thinking. There is a consensus among the researchers who conducted these studies, however, that maker activities and maker pedagogies go well beyond the teaching and learning of subject content, to the development of a wide variety of global skills and competencies, including creativity, digital literacy, critical thinking, collaboration, and communication. Makerspaces are also increasingly seen as spaces where makers can explore personal and cultural expression and reflection as a means of empowerment. In a mixed methods study on the impact of an Autism Inclusion Maker Program, Martin et al. found that students living with Autism Spectrum Disorder (ASD) who typically struggled in "normal" school settings were successful in creating their design-based projects and communicating with their peers about their projects, once they were freed from the constraints of typical classroom instruction. The authors found that the opportunity to create something that represented their personal interests was beneficial for both neurodivergent and neurotypical students. In an ethnographic study in a Finnish makerspace, researchers Iwata et al., explored to what extent global competencies, such as ways of thinking, ways of working, tools for working and ways of living in the world, are recognized and developed by teachers (pedagogy experts) and makerspace facilitators (technology experts) in digital making activities. Their findings suggest that engaging students in complex tasks that require frequent use of computers, student-centered facilitation and ill-structured activities, might enhance the development of global competencies and computational thinking practices; however, challenges around deep understandings of computational thinking and restricted time frames for making were evident in the study.

Four of the articles in this special issue shine a light on the role of teachers and facilitators in maker programs. US based researchers Bevan et al., explore how a research-practice partnership (RPP) might amplify the voices of informal educators leading afterschool maker programs related to science and suggest that these kinds of programs can complement school-based making by supporting student learning that is valued and relevant in school contexts. Bevan et al. Tinkering Design Framework, shared in their article, makes a significant contribution to the literature about making and tinkering, emphasizing the dialectical role that cultural tools and social contexts play in supporting the transformative agency of students. In Iceland, a seven-woman team of tech 
leaders and educators harnessed and asserted their transformative agency to support interested teachers in developing innovative pedagogies through digital technologies. Kjartansdottir et al. share the collective experiences of their team as they collaborated to bring about a cultural transformation in maker education, which has traditionally been dominated by white men. This article speaks to the importance of empowering girls' and women's digital literacies and design skills. Educational institutions have historically contributed to conditions of oppression and marginalization, but maker pedagogies have the potential to liberate when students are given opportunities to explore their personal and community identities and interests. In their design-based case study research in Canada, Becker and Jacobsen explore how one grade 6 teacher in a rural school, through collaboration with the authors, experienced a shift in pedagogical practice through three curricular making cycles focused on math and science. The teacher noticed that offering her students choice in both topics and the materials they used for model making led to growth in the flexibility of their thinking as well as their problem solving skills. The authors also note that making provided opportunities for the students to develop fundamental skills such as risk-taking, collaboration, communication and creativity. Also in Canada, Hughes and Morrison conducted a study involving 20 different elementary schools and 60 teachers, in which they examined the role of the makerspace itself as an actor in the learning process. Using case study methodology, they present findings from three makerspaces in particular, focusing on the locations, layouts, and tools selected for the spaces, and examined how these intersected to reflect the specific communities served by the makerspace. The authors note that the physical learning environment influences what kinds of learning can be facilitated there and how the spaces supported a transformation-based approach to pedagogy (different levels of inquiry) and therefore provided students with additional power in the learning process through increased collaboration, choice and control.

Kumpulainen et al. explore the cultural dimension of maker literacies, along with the critical and operational dimensions, in their ethnographic case study of students' maker literacies in a Finnish elementary school and its makerspace. Tracking the work of eleven elementary school students as they interacted with FUSE studio, the authors found that $74 \%$ of the students' multimodal interactions were operational, compared with $16 \%$ for the cultural dimension and $10 \%$ for the critical dimension. Their findings suggest that the cultural and critical dimensions of maker literacies need more attention in the making process. The cultural dimension refers to using, producing, and making sense of digital technologies and content in relation to cultural context, while the critical dimension refers to judging digital tools and digital content; identifying the intentions of designers and producers and how they position an audience; and considering issues of power, equity and diversity, persuasion, propaganda, and trust. The authors present a useful framework for the analysis of maker engagement across all three dimensions. This emphasis on the cultural context, which involves communicating and collaborating with digital tools and in digital environments while creating digital and media outputs was a key theme in several of the studies. In a research study conducted in a high school computer science class, Lindberg et al. explore what kinds of personal and community meanings students conveyed through art-focused maker projects. The authors found that arts-based STEAM practices such as conscious intent, consideration of audience, and conscious reflection through artistic communication supported their participation in communities both in and out of school and gave them the tools necessary to be able to represent or remediate their ideas to their peers and collective communities in which they belonged. This study, like others in this Research Topic, points to the role making can play in connecting makers to their communities through selfexpression.

Together, the articles of this Research Topic unpack the possibilities and challenges of maker education in diverse educational settings and among diverse participants. The articles not only illustrate how maker education can potentially transform education and learning but also how this often requires continuous collective efforts and cultural change.

\section{AUTHOR CONTRIBUTIONS}

All authors listed have made a substantial, direct, and intellectual contribution to the work and approved it for publication.

Conflict of Interest: The authors declare that the research was conducted in the absence of any commercial or financial relationships that could be construed as a potential conflict of interest.

Publisher's Note: All claims expressed in this article are solely those of the authors and do not necessarily represent those of their affiliated organizations, or those of the publisher, the editors and the reviewers. Any product that may be evaluated in this article, or claim that may be made by its manufacturer, is not guaranteed or endorsed by the publisher.

Copyright (c) 2021 Hughes and Kumpulainen. This is an open-access article distributed under the terms of the Creative Commons Attribution License (CC $B Y$ ). The use, distribution or reproduction in other forums is permitted, provided the original author(s) and the copyright owner(s) are credited and that the original publication in this journal is cited, in accordance with accepted academic practice. No use, distribution or reproduction is permitted which does not comply with these terms. 